\title{
Assimilation: central and peripheral effects
}

\author{
Charles M M de Weert, Noud A W H van Kruysbergen \\ Nijmegen Institute for Cognition and Information (NICl), University of Nijmegen, PO Box 9104, \\ 6500 HE Nijmegen, The Netherlands; e-mail: deweert@nici.kun.nl \\ Received 31 May 1996, in revised form 11 August 1997
}

\begin{abstract}
Assimilation and contrast have opposite effects: Contrast leads to an increase of perceived differences between neighbouring fields, whereas assimilation leads to a reduction. It is relatively easy to demonstrate these effects, but the precise localisation of these effects in the perceptual system is not yet possible. In an experiment the strength of assimilation effects was modified by adding spatial noise. By varying the localisation in perceived space of the added noise (by presentation of the noise pattern with different binocular disparities) the masking effect of this noise can be influenced. Masking caused by binocularly disparate noise is less than masking caused by binocularly non-disparate noise. It is concluded that the effect at least partly occurs beyond the (binocular) locus of separation in different depth planes. A similar approach, involving moving noise, is also presented. Finally, several demonstrations show that images that are peripherally similar can give rise to differences in the perceived amount of assimilation. These effects further indicate that a central mechanism is involved in assimilation.
\end{abstract}

\section{Introduction}

Assimilation is a perceived change in colour and/or brightness in one part of a picture in the direction of the colour and/or brightness of another part or other parts in the picture. The simplest case is a change in perceived colour of the background in the direction of the colour parts belonging to the foreground. Assimilation is more common in daily visual scenes than the better known simultaneous contrast effect (De Valois and De Valois 1988). Since the discovery of receptive fields with a centre-surround structure, lateral inhibition has been the most popular tool for explaining basic visual processes. Undoubtedly it is an important mechanism. The shape of the luminance contrast modulation transfer function can easily be understood on the basis of the mechanism of lateral inhibition, which in turn finds its physiological basis in spatial opponency of the centre-surround receptive fields.

Assimilation has been known for a long time (von Bezold 1876; Helson 1963). Kanizsa (1979) described a particular aspect of the assimilation effect, namely the influence of the observer's attitude. Assimilation effects are most distinct if the observer does not fixate steadily. In fact, he stated that an untrained observer clearly perceives assimilation, whereas a trained observer like a painter, is less certain whether contrast or assimilation occurs (Kanizsa was both a painter and a scholar of visual perception). Kanisza ascribed this difference to the distinction that trained observers make between the chromatic characteristics of objects and the nature of the illumination, and he supposed that the untrained observer completely ignores this distinction.

Festinger et al (1970) explained the occurrence of contrast or assimilation in terms of foreground/background perception: if part of a stimulus is considered as foreground, contrast occurs between this part and the background; but if the same part of the stimulus is seen as background, assimilation occurs within this part. This implies that the occurrence of assimilation does not precede figure-ground separation, or at least is dependent upon this separation to some extent. In the extreme case this means that assimilation only occurs after figure-ground separation, which is unlikely to be a retinal phenomenon. Another explanation is that the process of assimilation (and 
possibly also of contrast) is in principle a peripheral effect, but with strong feedback from a central level (Gove et al 1995). Indications for central effects pertaining to contrast phenomena have also been provided by De Valois and De Valois (1988). Sugita (1995) provides evidence that assimilation has a cortical origin, but provides no such evidence for contrast.

The neon spreading effect described by Cicerone et al (1995) also seems to point to the idea that figure-ground analysis (or more probably, in our view, a splitting of material and illumination properties) must have occurred before assimilation takes place, but not the other way round. In their case, the neon spreading effect (van Tuijl 1975; van Tuijl and de Weert 1979) occurs over a number of red dots in a surround of green dots, when the red of the dots moves as a spotlight over the dots, replacing the green colour of the dots in the background.

The notion of complex interactions occurring in the perception of illumination, material properties, and chromatic properties is strongly supported by Adelson (1993). It is rather unlikely that changes in appearance as demonstrated in Adelson's examples can be explained at a peripheral level.

In this paper we study possible central processing aspects of the assimilation effect. First, we describe the results of one simple experiment; subsequently we demonstrate a number of effects unlikely to have an exclusive peripheral basis.

The idea of the experiment and the demonstrations derive from a central theme: In some way the occurrence of assimilation and/or contrast is connected to the mechanism of attention, which is involved in the process of separating figure from ground. This separation is a central task for the visual system, and it is a complicated process because it can also entail separation of material and illumination properties.

The stimulus used in the experiment has been employed in many demonstrations and experiments (de Weert and van Kruysbergen 1987; de Weert 1991; de Weert and Spillmann 1995), and found to cause strong assimilation effects.

Figure la shows pincushion-like areas with identical levels of luminances. The area bounded by dark lines looks considerably darker than the area bounded by white lines.

By adding noise to the entire stimulus, we expect a reduced assimilation effect (see figure 1b). Suppose that this is the case (as will be tested in the experiment); then the following hypothesis can be put forward: If noise is perceptually separated from the stimulus prior to the level at which the assimilation effect originates, then the strength of the original effect should be regained.

Two methods can be used to present stimuli such that noise can be perceptually separated from the original stimulus. The first method is to separate noise by adding

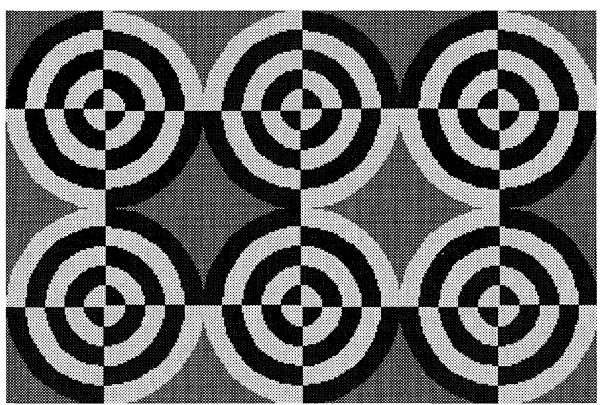

(a)

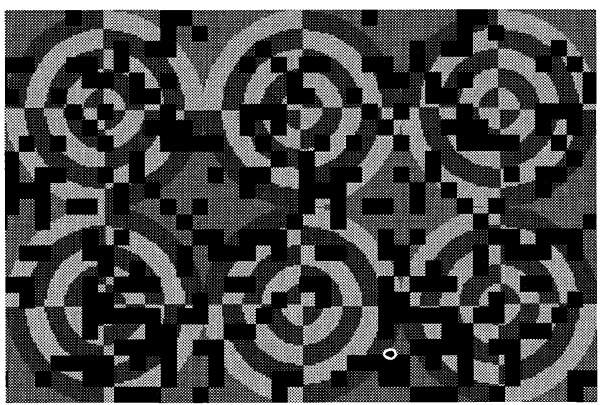

(b)

Figure 1. The stimulus used in the experiment. (a) The basic stimulus: The pincushions within the black borders look darker than the pincushions within the white borders, even though the luminances of the pincushions are the same. (b) The same stimulus with lower contrast and with noise added. 
identical noise patterns to the monocular test stimuli with some disparity. Binocular fusion of the two pictures can, with the proper disparity, result in a perceptual separation of the noise from the stimulus. Imagine that noise is added such that the noise appears in stereoscopic view as a layer in front of the assimilation stimulus. The question then is whether this perceptual separation gives rise to the perception of an assimilation pattern in the stimulus that is similar in strength to that of the undisturbed original stimulus.

The second method is to separate the noise patterns perceptually from the original stimulus by superimposing moving noise onto the stimulus. It is also possible to present the moving noise in another depth plane, in which case we expect the assimilation effect of the stimulus to be less disturbed than in the case where there is also disparity but with a static noise pattern.

\section{Experiment}

\subsection{Stimuli}

The stimulus shown in figure la is the basis of the stimuli used in this experiment. Three different levels of contrast were used. The luminance of the dark screen was $0.03 \mathrm{~cd} \mathrm{~m}^{-2}$; the luminance of the pincushions was $8.0 \mathrm{~cd} \mathrm{~m}^{-2}$; the luminances of the lines for the high-contrast stimulus were 14.8 and $3.1 \mathrm{~cd} \mathrm{~m}^{-2}$, for the medium-contrast stimulus 13.6 and $3.5 \mathrm{~cd} \mathrm{~m}^{-2}$, and for the low-contrast stimulus 12.4 and $4.1 \mathrm{~cd} \mathrm{~m}^{-2}$. The stimuli could contain random noise, as shown in figure $1 \mathrm{~b}$. The luminance of the noise was equal to the luminance of the dark screen.

The added noise was either static or dynamic (moving to the right). The noise could occur in one of three depth planes, including the same plane as the stimulus. This led to 21 possible stimuli.

An example of a trial is presented in figure 2. For convenience of the reader both crossed and uncrossed viewings are supported, but in our experiment only the former was employed. The left stimuli were presented to the left eye and the others to the right eye by using a septum. Thus, subjects always saw the stimuli dichoptically. The stimuli were presented on a Philips 15B monitor, run by an Atari TT030 in greyscale mode, in a darkened room. The visual angle of the stimulus was $10.9 \mathrm{deg} \times 7.4 \mathrm{deg}$. The disparity of level zero was 0 deg (noise in the same plane as the stimulus), the disparity of level one was $0.6 \mathrm{deg}$ and the disparity of level two was $1.2 \mathrm{deg}$. The size of the noise dots was $8 \times 8$ pixels $(0.27 \mathrm{deg})$. The amount of noise added was $30 \%$. For each position a random integer between 0 and 9 was drawn: If it was below 3 the stimulus was replaced by a black square, otherwise the original stimulus was shown on that spot. If the noise was moving, it moved with a velocity of $8.7 \mathrm{deg} \mathrm{s}^{-1}$.

\subsection{Subjects}

Three subjects participated in this experiment: the two authors and a third subject not involved in assimilation research.

\subsection{Method}

Subjects saw two stimuli, one at the top and one at the bottom of the screen. The task was to decide in which stimulus the brightness difference between the central pincushion on the left side and the central pincushion on the right side of the stimulus was larger. In case the effect appeared stronger in the top stimulus they had to press the up-arrow key and if the effect appeared stronger in the bottom stimulus they had to press the down-arrow key.

All 21 possible stimuli were presented pairwise, resulting in $21 \times 20 / 2=210$ trials in one session. These trials were randomised. Each subject took part in five sessions. The summed number of times that a particular stimulus gave rise to a stronger assimilation effect than any of the other stimulus types led to a rank order of the stimuli for that particular subject. 

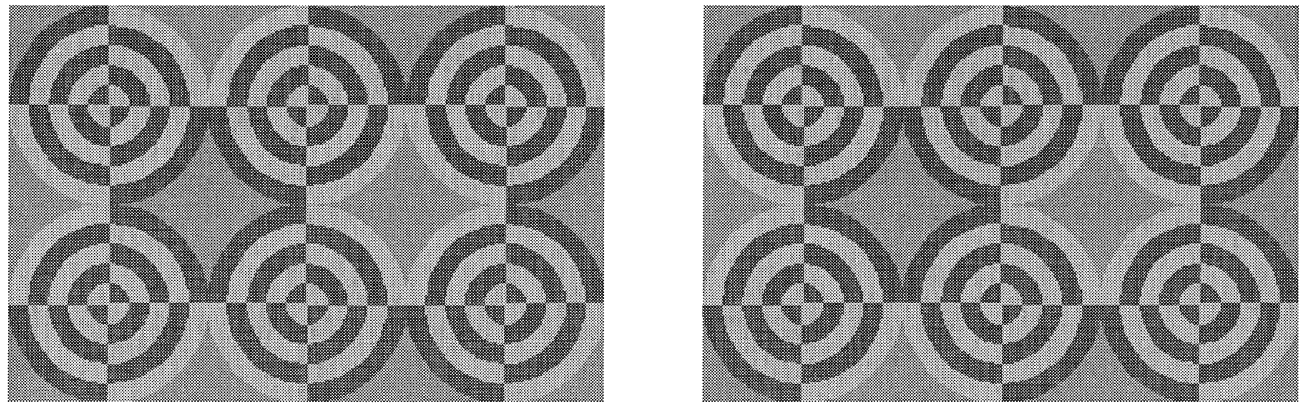

(a)
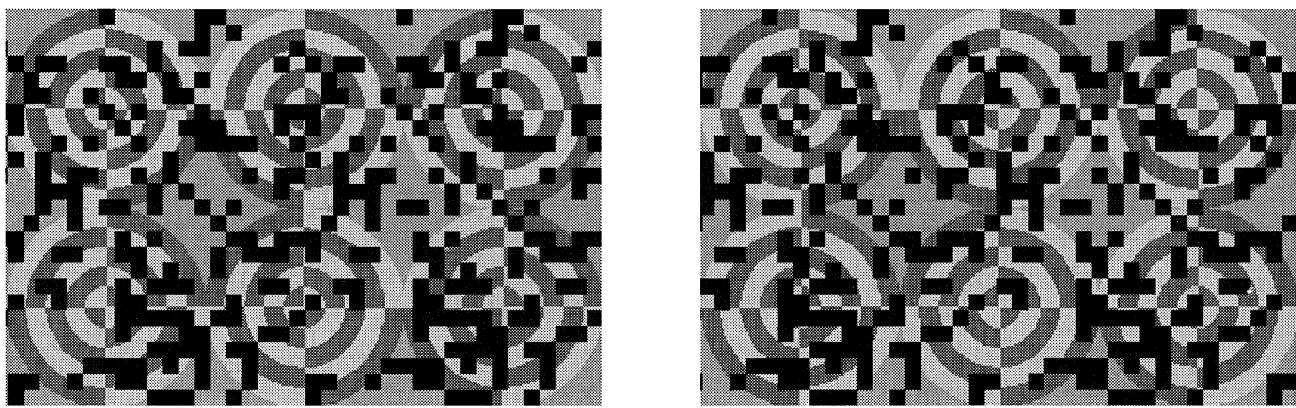

(b)
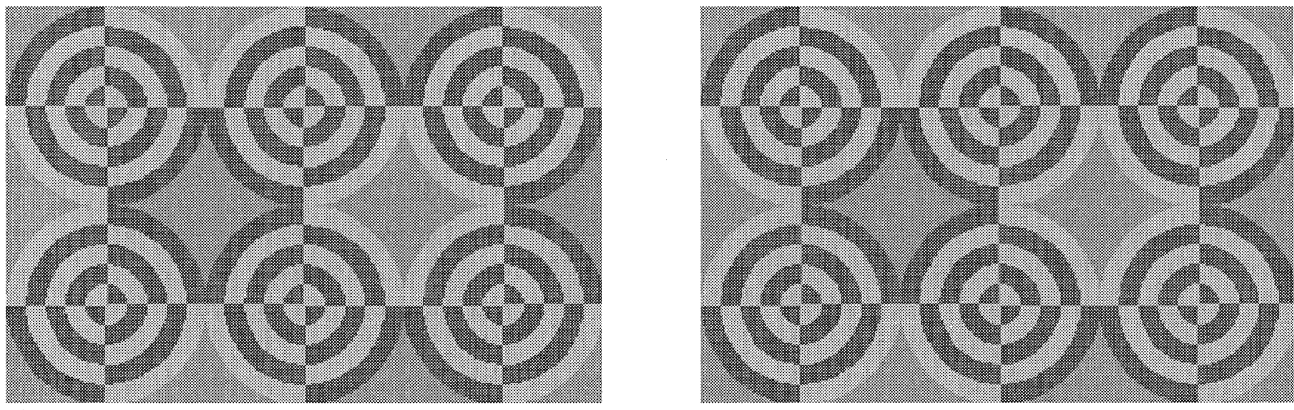

(c)
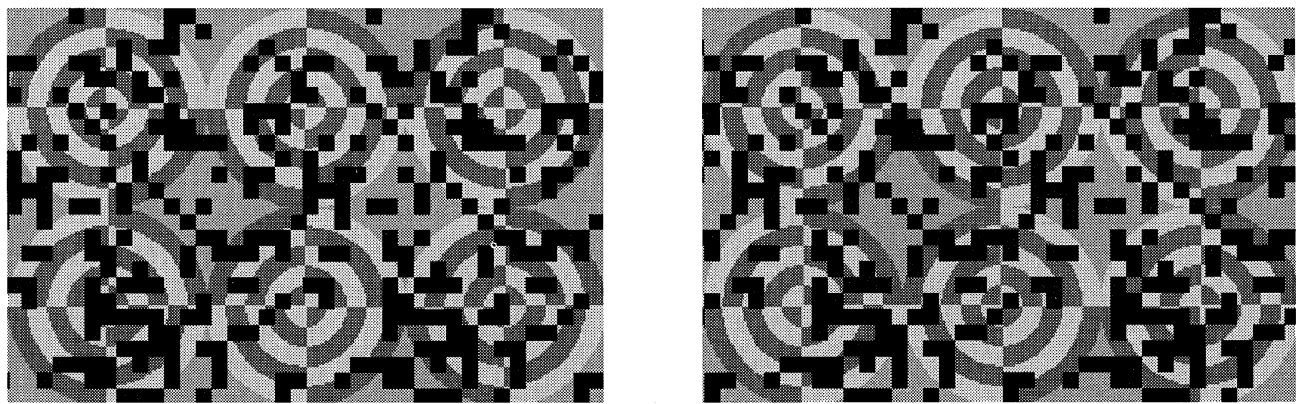

(d)

Figure 2. (a) and (c) A stimulus of middle contrast; (b) and (d) a stimulus of high contrast, with noise at depth plane 1. (a) and (b) are for uncrossed viewing; (c) and (d) are for crossed viewing. 


\subsection{Results}

Table 1 shows the resulting rank order of the stimuli. Kendall's coefficient of concordance ( $W=0.9479$ ) leads to a $\chi_{20}^{2}$ of 56.87 , which is highly significant $(p<0.001)$, indicating that the rank orders of the subjects are statistically the same.

As expected, within each contrast level the strongest effect is perceived in the undisturbed stimulus, whereas the weakest effect is obtained for the stimulus with stationary noise in the same depth plane. In between these two, the rank order roughly corresponds to the 'amount of evidence' for separation of figure and noise layer, either by depth or by motion or by both. Clearly, the effect of depth and motion together yields the strongest recovery of the original undisturbed effect. For all three contrast levels, the original undisturbed stimulus is the strongest, followed by the stimuli with the moving noise appearing in another depth plane. If stationary noise is used in another depth plane the perceived assimilation effect is larger than in the case when the noise is shown in the same depth plane, but it is smaller than in the case where the noise is moving. If moving noise is presented in the same depth plane there is still recovery. Only one violation of this general order occurs in the rank order of the highcontrast stimuli. In that case the moving noise in the same depth plane leads to a larger assimilation effect than stationary noise in a depth plane with the largest disparity.

Note that, for the rank order of each individual contrast level, separation of noise and figure by a small disparity leads to a stonger effect than when the noise and the figure are separated by a large disparity. Whether that is truly the case cannot be derived statistically from our data.

Table 1. The resulting rank order of the stimuli. Note that within each contrast level the original undisturbed stimulus is ranked as strongest, whereas the stimulus with static noise in the same plane is ranked as weakest.

\begin{tabular}{|c|c|c|c|c|c|c|c|}
\hline $\begin{array}{l}\text { rank } \\
\text { order }\end{array}$ & $\begin{array}{l}\text { high } \\
\text { contrast }\end{array}$ & $\begin{array}{l}\text { medium } \\
\text { contrast }\end{array}$ & $\begin{array}{l}\text { low } \\
\text { contrast }\end{array}$ & $\begin{array}{l}\text { rank } \\
\text { order }\end{array}$ & $\begin{array}{l}\text { high } \\
\text { contrast }\end{array}$ & $\begin{array}{l}\text { medium } \\
\text { contrast }\end{array}$ & $\begin{array}{l}\text { low } \\
\text { contrast }\end{array}$ \\
\hline 1 & \% & & & 12 & & & 政 \\
\hline 2 & & 80 & & 13 & & & (2) \\
\hline 3 & 愈列 & & & 14 & & 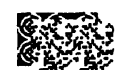 & \\
\hline 4 & ram & & & 15 & 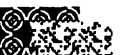 & & \\
\hline 5 & & & (2) & 16 & & ast & \\
\hline 6 & & 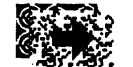 & & 17 & & 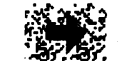 & \\
\hline 7 & & (3) & & 18 & & & 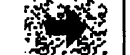 \\
\hline 8 & & & 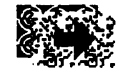 & 19 & & 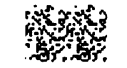 & \\
\hline 9 & & & 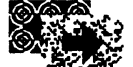 & 20 & 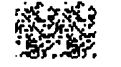 & & \\
\hline 10 & 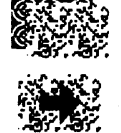 & & & 21 & & & 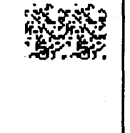 \\
\hline
\end{tabular}




\section{Discussion}

The results clearly support the idea that separation of figure and noise leads to a recovery of the strength of the assimilation effect.

The second part of this paper has the character of a set of demonstrations rather than of a real experiment. The main aim is again to try to separate parts of a figure, either through stereo or through other methods; and to test whether separation of figure and ground leads to an observable change in assimilation.

In figure 3 the red and the green dots are seen in different depth planes. In the upper part the red dots are perceived in the background plane, in the lower part the green dots are perceived in the background (or the reverse depending upon the disparity). All five subjects clearly and systematically saw the colours of the background as different in the two cases: In the upper stereogram the plane in the background was perceived greenish, and in the lower stereogram the background plane was perceived as more reddish than the surround. ${ }^{(1)}$ Apparently, the observed assimilation corresponds to the assimilation occurring in the background plane.

An interesting stimulus producing a change in appearance within a single plane is presented in figure 4. Here the 'red' plane and the 'green' plane cross in depth along the vertical midline, and with this crossing the appearance of the background changes.

A similar effect may be observed in figure 5. With careful inspection of one of the monocular stimuli, a similar splitting of the depth planes and even a reversal can be seen. Again, subjects observed a change in appearance of the perceived background

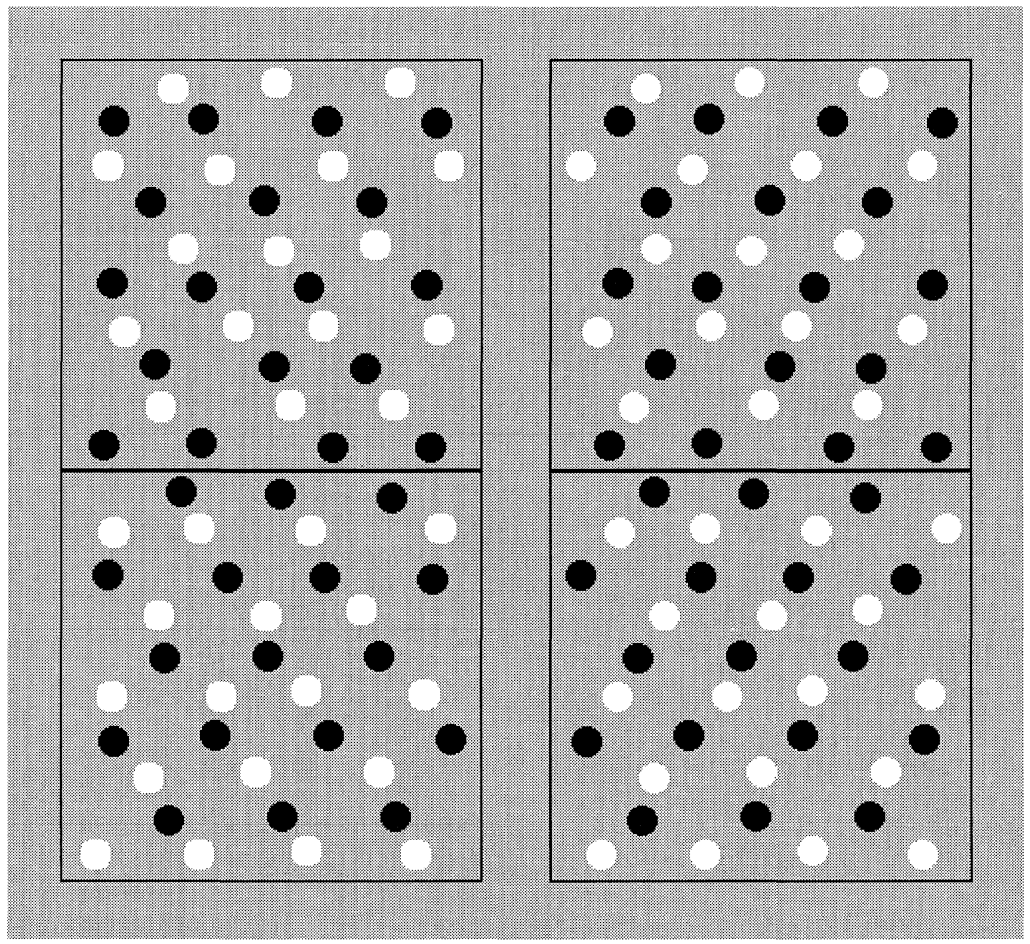

Figure 3. Left and right parts have to be combined dichoptically to give rise to two depth planes, one filled with red dots and one filled with green dots (for the achromatic version: white dots and black dots). Where the green (black) dots are seen in the background the perceived assimilation effect is greenish (darker), whereas there is reddish assimilation (brighter) where the red (white) dots are seen in the background.

(1) Colour versions of the figures are available at http://www.pion.co.uk/perception/perc1097/ deweert.html 

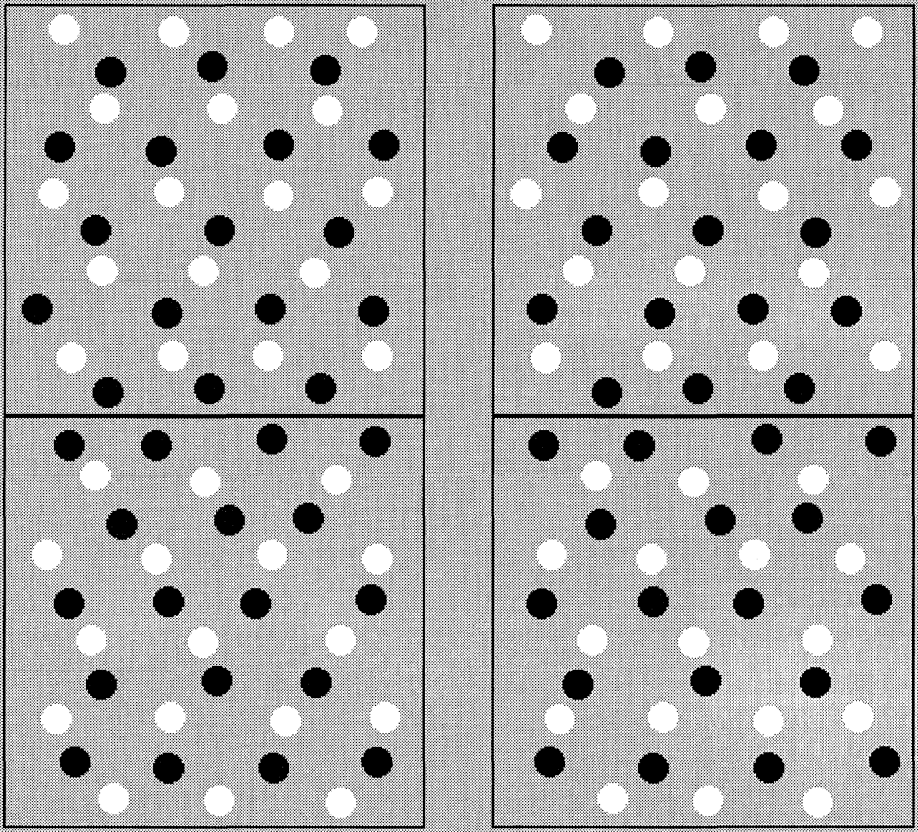

Figure 4. This figure, when viewed stereoscopically, gives rise to two planes crossing in depth at the vertical midline.

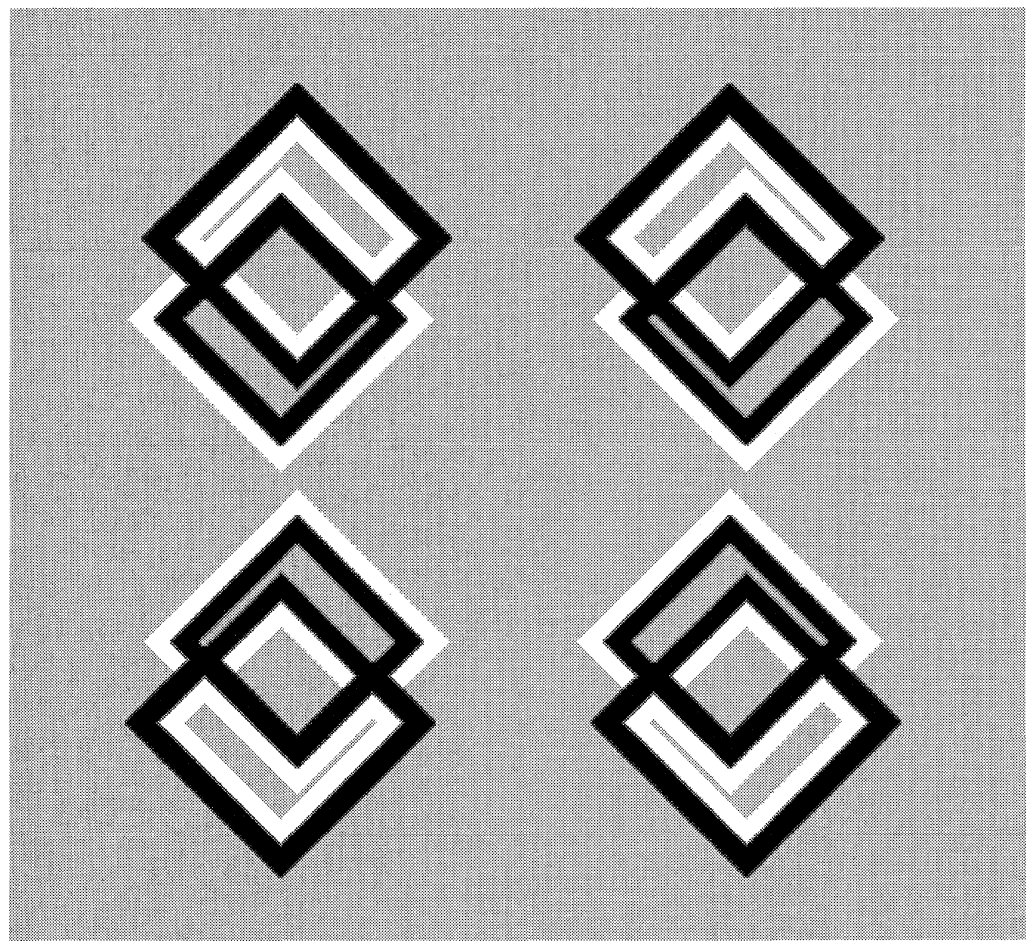

Figure 5. Stereoscopic combination leads to the perception of two figures in depth and to different patterns of assimilation. 
figure if the percept of the red-lined figure ("background looks reddish") alternated with the percept of the green-lined figure ("background looks greenish"); or, in case of the achromatic stimulus, if the percept of the white-lined figure ("background looks brighter") alternated with the percept of the black-lined figure ("background looks darker").

We conclude that assimilation cannot simply be a local retinal effect. In previous papers (de Weert and van Kruysbergen 1987; de Weert 1991) it was argued that assimilation effects were based on local averaging of colour and/or luminance within large receptive fields. This cannot be maintained any longer, as the experiments clearly show that assimilation occurs after separation of the picture into figure and ground, or at least after separation into different depth planes.

The demonstrations presented in this study imply that whatever the precise nature of the assimilation process, it cannot be a pure bottom-up retinal process.

Acknowledgments. The authors are very thankful to Marco Puts and Peter Snoeren, PhD students at NICI, for their valuable contributions to the discussions, and for their willingness to take part in pilot studies and to produce new stimuli again and again. Intensive discussions with the editors have also strongly improved this paper.

Part of the data reported in this paper has been presented at the 18th ECVP, Tübingen, 1995.

\section{References}

Adelson E, 1993 "Perceptual organization and the judgment of brightness" Science 262 2042-2044

Bezold von W, 1876, in Evans R M An Introduction to Color (1948, New York: John Wiley), page 181

Cicerone C, Hoffman D, Gowdy P D, Kim J S, 1995 "The perception of color from motion" Perception \& Psychophysics $57761-777$

De Valois R L, De Valois K, 1988 Spatial Vision (New York: Oxford University Press)

Festinger L, Coren S, Rivers G, 1970 "The effect of attention on brightness contrast and assimilation" American Journal of Psychology 83 189-207

Gove A, Grossberg S, Mingolla E, 1995 "Brightness perception, illusory contours, and corticogeniculate feedback" Visual Neuroscience 12 1027-1052

Helson H, 1963 "Studies of anomalous contrast and assimilation" Journal of the Optical Society of America 53179 - 184

Kanizsa G, 1979 Organization in Vision. Essays on Gestalt Perception (New York: Praeger)

Sugita Y, 1995 "Contrast and assimilation on different depth planes" Vision Research 35 881-884

Tuijl H F J M van, 1975 "A new visual illusion: neonlike color spreading and complementary color induction between subjective contours" Acta Psychologica 39441 - 445

Tuijl H F J M van, Weert Ch M M de, 1979 "Sensory conditions for the occurrence of the neon spreading illusion" Perception $8211-215$

Weert Ch M M de, 1991 "Assimilation versus contrast", in From Pigments to Perception Eds A Valberg, B B Lee (New York: Plenum) pp 305-311

Weert Ch M M de, Kruysbergen A W H van, 1987 "Strength of subjective contours and perceived superimposition: transparency as a special case", in Perception of Illusory Contours Eds S Petry, G Meyer (New York: Springer) pp 165-170

Weert Ch M M de, Spillmann L, 1995 "Assimilation: asymmetry between brightness and darkness" Vision Research 35 1413-1420 